\title{
Transoral incisionless fundoplication with EsophyX for gastro- esophageal reflux disease: clinical efficacy is maintained up to 10 years
}

(1) $\odot$

\author{
Authors \\ Pier Alberto Testoni, Sabrina Testoni, Giovanni Distefano, Giorgia Mazzoleni, Lorella Fanti, Sandro Passaretti
}

Institution

Division of Gastroenterology and Gastrointestinal Endoscopy, Vita-Salute San Raffaele University, San Raffaele Scientific Institute, Milan, Italy

submitted 31.8 .2018

accepted after revision $\quad 18.10 .2018$

Bibliography

DOI https://doi.org/10.1055/a-0820-2297 |

Endoscopy International Open 2019; 07: E647-E654

(c) Georg Thieme Verlag KG Stuttgart · New York elSSN 2196-9736

Corresponding author

Pier Alberto Testoni, MD, Division of Gastroenterology and Gastrointestinal Endoscopy, Vita-Salute San Raffaele University, San Raffaele Scientific Institute, Via Olgettina 58, 20132 Milan, Italy

Fax: +39-2-26433491

testoni.pieralberto@hsr.it

\section{ABSTRACT}

Background Transoral incisionless fundoplication with EsophyX is reported to be effective in patients with gastroesophageal reflux disease in short-medium term follow-up.
Aim To examine clinical outcomes up to 10 years. Methods In total, 51 procedures were performed in 50 patients. All entered a yearly clinical follow-up schedule including gastroesophageal reflux disease health-related quality-of-life questionnaires, heartburn and regurgitation scores, and daily proton pump inhibitor consumption.

Results The procedure was successfully performed in 49/ 50 patients. Severe complications occurred in 2/51 procedures. The remaining 49 patients were re-evaluated at 2 and 3 years, 41 after 5 years, 30 after 7 years, and 14 after 10 years. Eight patients were lost to follow-up between 3 and 5 years. Seven patients who were unresponsive to endoscopic fundoplication underwent surgical fundoplication. The mean scores at 2 years were significantly lower than before the procedure and did not change substantially during the follow-up. The rates of patients who had stopped or halved antisecretive therapy $2,3,5,7$, and 10 years after the procedure were $86.7 \%, 84.4 \%, 73.5 \%, 83.3 \%$, and $91.7 \%$, respectively.

Conclusions Transoral incisionless fundoplication with EsophyX is an effective therapeutic option for symptomatic gastroesophageal reflux disease patients, with Hill grades I-II or hiatal hernia $<2 \mathrm{~cm}$, who refuse life-long medical therapy or surgery.

\section{Introduction}

Transoral incisionless fundoplication (TIF) has become a widely used intervention to restore the valve at the gastroesophageal junction in selected cases suffering from gastroesophageal reflux disease (GERD). More than 20000 procedures have been done so far, as reported by the manufacturer of EsophyX (EndoGastric Solutions, Redmond, WA, United States) - the most widely used device. TIF with the EsophyX device reconfigures the tissue to establish an omega-shaped, full-thickness gastroesophageal valve from inside the stomach. The procedure creates serosa-to-serosa plications which include the muscle layers and constructs valves $3-5 \mathrm{~cm}$ long, taking in $200-270^{\circ}$ of the circumference, and deploying multiple non-absorbable polypropylene fasteners through the two layers in a circumferential pattern around the gastroesophageal junction [1-4].

Data so far indicate that, in the majority of patients, the procedure achieves lasting improvement in GERD symptoms, with cessation or reduction in proton pump inhibitor (PPI) therapy and improvement in functional findings, measured by either $\mathrm{pH}$ or impedance monitoring.

The technique offers an acceptable alternative to surgery, mimicking partial anterior fundoplication, but less invasive and with no persistent side effects, in patients with proven pathological GERD with either a competent gastroesophageal valve or hiatal hernia not longer than $3 \mathrm{~cm}$, who refuse, are in- 
tolerant, or are unresponsive to PPI maintenance therapy. Laparoscopic fundoplication, although still considered to be the gold-standard approach for GERD refractory to medical treatment, does involve some risk of long-term adverse events such as dysphagia (5-12\%), inability to vomit or belch, gas/bloat syndrome (19\%), and excessive flatulence [5-7].

Several prospective observational studies and some comparative trials have proved the efficacy of TIF with EsophyX in obtaining a significant reduction in the acid exposure time (AET) assessed by esophageal 24-hour $\mathrm{pH}$-impedance monitoring versus sham, and in controlling both typical and atypical GERD symptoms for up to 1 and 2 years, as reported in a recent meta-analysis [8]; however, only six have studied the outcomes at $3[9-12]$ and 5 years $[13,14]$, and just one listed results at 5 to 6 years in a limited number of patients [11]. The relatively short follow-up in patients treated with TIF compared with surgery has raised concerns about the long-term efficacy of the procedure [15].

This study therefore examined the clinical efficacy of TIF at $2,3,5,7$, and 10 years in a series of patients who had undergone TIF 2.0 with the EsophyX device, performed by a single operator.

\section{Materials and methods}

In total, 50 patients underwent TIF 2.0 for symptomatic GERD, according to the Rome III criteria [16], from January 2007 to December 2012, and were scheduled for yearly follow-up for up to 10 years in a single-center observational prospective study. Clinical outcomes at 2, 3, 5, 7, and 10 years after the intervention were the subject of the present study.

The indication for TIF in all but two patients was pathological gastroesophageal reflux (GER) with a positive correlation between symptoms and GER, documented by 24-hour $\mathrm{pH}$-impedance. Two patients had 24-hour $\mathrm{pH}$-impedance in the normal range but a positive correlation between symptoms and GER (hypersensitive esophagus). All but two of the patients were responsive or partially responsive to PPI. All patients complained of heartburn and/or regurgitation and had been on PPI maintenance therapy with a standard dose twice a day for at least 3 months before enrollment. For each patient, clinical and procedural data were stored in a computerized database in the endoscopy unit.

Patients with atypical symptoms of GERD, functional reflux, hiatal hernia longer than $3 \mathrm{~cm}$, Barrett's esophagus, esophageal stricture, previous esophageal, gastric or major abdominal surgery, and other severe comorbidities (including collagen disease) were excluded from the study.

Fundoplication (TIF 2.0) was performed in all cases by a single endoscopist with in vivo experience in animal models and humans.

All patients gave written informed consent for the procedure and for data management for scientific purposes. Both the enrollment protocol and the long-term clinical assessment were approved by the Medical Ethics Committee of San Raffaele Scientific Institute of Milan. The study protocol conforms to the ethical guidelines of the 1975 Declaration of Helsinki (6th revi- sion, 2008) as reflected in a priori approval by the institution's human research committee.

\section{Study protocol}

At enrollment, all patients completed the Gastroesophageal Reflux Disease Health-Related Quality-of-Life (GERD-HRQL) and Gastroesophageal Reflux Disease Quality-of-Life (GERDQUAL) questionnaires while on a standard dose of PPI twice a day. PPI was then stopped for 14 days and the patients were asked to complete the two questionnaires once again. Heartburn and regurgitation scores were assessed separately. The GERD-HRQL is a validated 16-item questionnaire that measures the symptom severity of GERD patients [17]. Six items measure satisfaction with the degree of heartburn, two satisfaction with dysphagia/pain while swallowing, and one satisfaction with the impact of medication on daily life; one item measures overall satisfaction with the present condition, and six items measure satisfaction with regurgitation. Each item is scored from 0 to 5 . Heartburn and regurgitation scores were extrapolated from the GERD-HRQL questionnaire and assessed separately. The GERD-QUAL is a validated 37-item questionnaire that measures the quality of life of GERD patients [18]; each item is scored from 1 to 5 .

Patients then underwent:

- upper gastrointestinal endoscopy to determine the Hill grade and Jobe length of the gastroesophageal valve; the presence and size of hiatal hernia; the presence and severity of esophagitis according to the Los Angeles grading system [19];

- stationary esophageal manometry and 24-hour ambulatory $\mathrm{pH}$-impedance monitoring performed off PPI (PPI therapy suspended at least 12 days previously); any drugs influencing gastrointestinal motility were discontinued 14 days before the functional investigation;

- scintigraphic recording of gastric emptying time.

GERD-HRQL and GERD-QUAL questionnaires, heartburn and regurgitation scores, daily PPI consumption, upper gastrointestinal endoscopy, esophageal manometry, and 24-hour ambulatory $\mathrm{pH}$-impedance were repeated 6,12 , and 24 months after the TIF. Ambulatory $\mathrm{pH}$-impedance was recorded when off PPI in patients who had still been taking them. GERD-QUAL was no longer assessed after 2 years.

GERD-HRQL, heartburn and regurgitation scores, and daily PPI consumption were investigated by telephone interview or office consultation 3, 5, 7, and 10 years after TIF.

All patients were contacted, including those unresponsive to TIF and who had undergone surgery. Two independent physicians unaware of the endoscopic procedures, initial post-interventional 2-year follow-up, and esophageal motility, conducted the long-term surveillance.

PPI consumption was considered "continued" when the daily drug dose was the same as before the procedure; "reduced" when any daily dose was taken for less than half the total number of days during follow-up; and "completely stopped" when not one dose of PPI was taken during the follow-up. 
- Table 1 Baseline clinical, functional and endoscopic characteristics of patients $(n=50)$ who underwent Transoral Incisionless Fundoplication 2.0 with EsophyX device.

\begin{tabular}{|l|c|}
\hline Sex, $\mathrm{n}(\%)$ & $35 / 50(70)$ \\
\hline - Male & $15 / 50(30)$ \\
\hline - Female & $45 \pm 16$ \\
\hline Age, mean \pm SD, years & $22 \pm 3$ \\
\hline Body Mass Index, mean \pm SD, kg/m² & \\
\hline GERD-HRQL score, mean \pm SD & $20 \pm 13$ \\
\hline - On PPI & $46 \pm 19$ \\
\hline - Off PPI ${ }^{2}$ & $18 \pm 9$ \\
\hline Heartburn score, mean \pm SD & $17 \pm 9$ \\
\hline Regurgitation score, mean \pm SD & \\
\hline Response to standard dose of PPI twice a day, $\mathrm{n}(\%)$ & $36 / 50(72)$ \\
\hline - Completely responsive & $12 / 50(24)$ \\
\hline - Partially responsive ${ }^{2}$ & $2 / 50(4)$ \\
\hline - Not responsive & $28 / 50(56)$ \\
\hline Hiatal hernia, n (\%) & $26 / 28(92.8)$ \\
\hline - 1 - 2 cm long & $1 / 28(3.6)$ \\
\hline - 2.5 cm long & $1 / 28(3.6)$ \\
\hline - 3 cm long & $11 / 50(22)$ \\
\hline Esophagitis (Los Angeles classification), $\mathrm{n}(\%)$ & $1 / 11(90.9)$ \\
\hline - Grade A & \\
\hline - Grade B & \\
\hline & \\
\hline
\end{tabular}

Hill's grade of gastroesophageal valve, $n$ (\%)

- Gradel 3/50(6)

- Grade II $34 / 50(68)$

\begin{tabular}{|l|c|}
\hline -Grade III & $12 / 50(24)$ \\
\hline - Grade IV & $1 / 50(2)$ \\
\hline $\begin{array}{l}\text { Jobe's length of gastroesophageal valve, } \\
\text { mean } \pm \text { SD, cm }\end{array}$ & $0.98 \pm 0.5$ \\
\hline \begin{tabular}{l} 
Ineffective esophageal motility, n (\%) \\
\hline Gastric emptying time abnormally long, n (\%)
\end{tabular} & $18 / 50(36)$ \\
\hline
\end{tabular}

n, number; SD, standard deviation; GERD-HRQL, Gastro-Esophageal Reflux Disease-Health-Related Quality-of-Life; PPI, proton pump inhibitors.

1 Off PPI therapy: patients discontinued PPI for at least 14 days before enrollment. $\mathrm{P}<0.01$ off PPI vs on PPI.

2 Partially responsive: defined as a GERD-HRQL score $>12$ on standard dose twice a day for at least 4 weeks.

\section{Statistical analysis}

Intra- and inter-patient characteristics, GERD-HRLQ and GERDQUAL total scores, heartburn and regurgitation scores, morphological and functional findings were compared by Wilcoxon's and Mann-Whitney tests or Fisher's exact test, as appropriate. A $P$ value $<0.05$ was considered to be statistically signifi-
- Table 2 Technical data for Transoral Incisionless Fundoplication 2.0 procedures $(n=51)$ with EsophyX device.

Technical success, $\mathrm{n}(\%)$

$49 / 51(96.1)$

Technical failure, $\mathrm{n}(\%)$

2/51(3.9)

- Intraprocedural pneumothorax

$1 / 2(50)$

- Device malfunction (TIF 2.0 repeated with suc-

$1 / 2(50)$ cess)

\begin{tabular}{|c|c|}
\hline $\begin{array}{l}\text { Fasteners deployed to construct each valve, } \\
\text { mean } \pm S D, n\end{array}$ & $12 \pm 4$ \\
\hline Post-TIF 2.0 hiatal hernia, n (\%) & 0 \\
\hline Hill's grade I for newly created valve, n (\%) & $49 / 51(96.1)$ \\
\hline Jobe's length of newly created valve, mean \pm SD, $\mathrm{cm}^{1}$ & $2.7 \pm 0.4$ \\
\hline Post-procedure severe complications, n (\%) & \\
\hline - Pneumothorax & $2(3.9)$ \\
\hline
\end{tabular}

cant. Data are presented as mean \pm SD. Per-protocol and intention-to-treat analysis were performed; the latter include patients lost to follow-up.

\section{Results}

The patients' main clinical and endoscopic characteristics are reported in - Table 1. TIF 2.0 was successful in 49/50 patients. In two cases, the procedure was interrupted: in one case on account of pneumothorax, in the other for device malfunction. The latter procedure was repeated with success. In all, 51 TIF procedures were performed in 50 patients.

A mean of $12 \pm 4$ fasteners were deployed to construct each valve. Hiatal hernias were always reduced. In all cases, the Hill's grade of the newly created valve was I, and its mean length was statistically significantly greater than before the procedure ( $2.7 \pm 0.4$ vs. $0.98 \pm 0.5 \mathrm{~cm} ; P<0.01)$.

Severe complications arose in two of the 51 procedures (3.9\%), both pneumothorax. These were confirmed by X-ray immediately after the procedure, and managed by immediate transthoracic drainage. Both patients had rapid resolution of the pneumothorax and were discharged from hospital within 3 days. No persistent post-intervention side effects were reported.

Data are summarized in > Table 2. Post-TIF endoscopic and functional findings have already been published [11], and are not the subject of the present clinical follow-up report.

All 49 patients were clinically evaluated at 2 and 3 years, 41 (83.7\%) after 5 years, 30 (61.2\%) after 7 years, and 14 (28.6\%) after 10 years. Eight patients were lost to follow-up between 3 and 5 years; no other patients were lost during the subsequent follow-up.

Data on follow-up of patients and clinical efficacy of TIF assessed by GERD-HRQL, heartburn and regurgitation scores, and PPI consumption at 2, 3, 5, 7, and 10 years, according to 
- Table 3 Data on follow-up and PPI use in patients $(n=49)$ who underwent Transoral Incisionless Fundoplication 2.0 with EsophyX device and its clinical efficacy according to per-protocol analysis (excluding patients who underwent Nissen fundoplication and those lost to follow-up) and intention-to-treat analysis (including patients who underwent procedure-related complications and Nissen fundoplication and excluding those lost to follow-up).

\begin{tabular}{|c|c|c|c|c|c|}
\hline & 2 years & 3 years & 5 years & 7 years & 10 years \\
\hline Patients on follow-up, n (\%) & $45 / 49(91.8)$ & $45 / 49(91.8)$ & $34 / 49(69.4)$ & $24 / 49$ (49) & $12 / 49(24.5)$ \\
\hline $\begin{array}{l}\text { Patients who underwent surgical fundoplication } \\
\text { on follow-up, } \mathrm{n}(\%)\end{array}$ & $4 / 49(8.2)$ & $4 / 49(8.2)$ & $7 / 49(14.3)$ & $6 / 49(12.2)$ & $2 / 49(4.1)$ \\
\hline Patients lost to follow-up, n (\%) & 0 & 0 & $8 / 49(16.3)$ & $5 / 49(10.2)$ & $5 / 49(10.2)$ \\
\hline \multicolumn{6}{|l|}{ Clinical success, $n(\%)^{1}$} \\
\hline - Per-protocol analysis & $39 / 45(86.7)$ & $38 / 45(84.4)$ & $25 / 34(73.5)$ & $20 / 24(83.3)$ & $11 / 12(91.7)$ \\
\hline - Intention-to-treat analysis & $39 / 50(78)$ & $38 / 50(76)$ & $25 / 42(59.5)$ & $20 / 31(64.5)$ & $11 / 15(73.3)$ \\
\hline \multicolumn{6}{|l|}{ Clinical failure, $\mathrm{n}(\%)^{2}$} \\
\hline - Per-protocol analysis & $6 / 45(13.3)$ & $7 / 45(15.6)$ & $9 / 34(26.5)$ & $4 / 24(16.7)$ & $1 / 12(8.3)$ \\
\hline - Intention-to-treat analysis & $6 / 50(12)$ & $7 / 50(14)$ & $9 / 42(21.4)$ & $4 / 31(12.9)$ & $1 / 15(6.7)$ \\
\hline \multicolumn{6}{|l|}{ Stopped PPI } \\
\hline - PP analysis & $25 / 45(55.6)$ & $24 / 45(53.3)$ & $14 / 34(41.2)$ & $11 / 24(45.8)$ & $5 / 12(41.7)$ \\
\hline - ITT analysis & $25 / 50(50)$ & $24 / 50(48)$ & $14 / 42(33.3)$ & $11 / 31(35.5)$ & $5 / 15(33.3)$ \\
\hline \multicolumn{6}{|l|}{ Halved PPI } \\
\hline - PP analysis & $14 / 45(31.1)$ & $14 / 45(31.1)$ & $11 / 34(32.3)$ & $9 / 24(37.5)$ & $6 / 12(50)$ \\
\hline - ITT analysis & $14 / 50(28)$ & $14 / 50(28)$ & $11 / 42(26.1)$ & $9 / 31(29)$ & $6 / 15(40)$ \\
\hline \multicolumn{6}{|l|}{ Unchanged PPI } \\
\hline - PP analysis & $6 / 45(13.3)$ & $7 / 45(15.6)$ & $9 / 34(26.5)$ & $4 / 24(16.7)$ & $1 / 12(8.3)$ \\
\hline - ITT analysis & $6 / 50(12)$ & $6 / 50(12)$ & $9 / 42(21.4)$ & $4 / 31(12.9)$ & $2 / 15(13.3)$ \\
\hline \multicolumn{6}{|l|}{ Stopped or halved PPI } \\
\hline - PP analysis & $39 / 45(86.7)$ & $38 / 45(84.4)$ & $25 / 34(73.5)$ & $20 / 24(83.3)$ & $11 / 12(91.7)$ \\
\hline - ITT analysis & $39 / 50(78)$ & $38 / 50(76)$ & $25 / 42(59.5)$ & $20 / 31(64.5)$ & $11 / 15(73.3)$ \\
\hline
\end{tabular}

per-protocol and intention-to-treat analysis, are summarized in - Table 3, - Table4. Seven patients unresponsive to TIF underwent Nissen fundoplication during the 10-year follow-up (14.3 $\%$ ): four in the first year, three between 3 and 5 years after TIF. One of the first four patients was lost at the 7-year follow-up (not contactable). In the subsequent years, no further patients underwent surgery. All patients who required surgery within 12 months after TIF had preoperative Hill's grade III or more for the valve.

Over the 10-year follow-up, the GERD-HRQL scores when off PPI therapy, and heartburn and regurgitation scores remained significantly lower than the pre-TIF scores. The reduction was significant at 2 years and scores were similar at 3 years; between 3 and 5 years, there was a further slight reduction in the mean scores; at 7 and 10 years, scores did not change substantially from the 5-year picture. Mean and median GERDHRQL heartburn and regurgitation scores appeared substantial- ly unchanged between 2 and 10 years after the TIF procedure ( Fig. 1, $>$ Table 4).

The percentages of patients who had stopped or halved the PPI therapy 2, 3, 5, 7, and 10 years after TIF were $86.7 \%, 84.4 \%$, $73.5 \%, 83.3 \%$, and $91.7 \%$, respectively; the rates remained substantially stable and similar to those at 2 years, with a tendency toward improvement at 10 years.

Considering only patients who completely stopped PPI therapy to be responders to TIF, there were $55.6 \%, 53.3 \%, 41.2 \%$, $45.8 \%$, and $41.7 \%$ full responders at $2,3,5,7$, and 10 years, respectively. The complete response rate after TIF had fallen by about $20 \%$ at 10 years compared to that at 2 years but the differences were not statistically significant $(P>0.05)(\nabla$ Fig.2a,

\section{- Table 3).}

Intention-to-treat analysis of the effect of TIF on PPI use, including all 50 patients scheduled to undergo the procedure, showed that at $2,3,5,7$, and 10 years after TIF, respectively, 
- Table4 Data on symptom scores during follow-up after Transoral Incisionless Fundoplication by EsophyX.

\begin{tabular}{|c|c|c|c|c|c|c|c|c|c|c|c|}
\hline & Pre & 2 years & $P$ vs pre & 3 years & $P$ vs pre & 5 years & $P$ vs pre & 7 years & $P$ vs pre & 10 years & $P$ vs pre \\
\hline No. of patients & 49 & 45 & & 45 & & 34 & & 24 & & 12 & \\
\hline $\begin{array}{l}\text { GERD-HRQL score } \\
\text { (off PPI), mean } \pm \text { SD }\end{array}$ & $46 \pm 19$ & $18 \pm 13$ & $<0.01$ & $19 \pm 14$ & $<0.01$ & $10 \pm 7$ & $<0.001$ & $10 \pm 7.7$ & $<0.001$ & $9.5 \pm 6.1$ & $<0.001$ \\
\hline $\begin{array}{l}\text { Heartburn score, } \\
\text { mean } \pm S D\end{array}$ & $18 \pm 9$ & $8 \pm 7$ & $<0.01$ & $9 \pm 8$ & $<0.01$ & $4.5 \pm 4.6$ & $<0.01$ & $4.6 \pm 4.7$ & $<0.01$ & $4.2 \pm 3$ & $<0.01$ \\
\hline $\begin{array}{l}\text { Regurgitation score, } \\
\text { mean } \pm \text { SD }\end{array}$ & $17 \pm 9$ & $9 \pm 6$ & $<0.01$ & $10 \pm 6$ & $<0.01$ & $3.2 \pm 4.3$ & $<0.01$ & $3.3 \pm 4.4$ & $<0.01$ & $3.2 \pm 4.4$ & $<0.01$ \\
\hline
\end{tabular}

$78 \%, 76 \%, 59.5 \%, 64.5 \%$, and $73.3 \%$ of patients had stopped or halved PPI therapy, and 50\%, 48\%, 33.3\%, 35.5\%, and $33.3 \%$ had completely discontinued it. The rate of patients who stopped or halved their dose of PPI did not change over time, while those who completely stopped PPI decreased by about $40 \%$ in the 10 years ( $\vee$ Table 3 ).

As regards procedure-related complications, for patients still taking the same dose of PPI as before TIF, and those who underwent surgical fundoplication, TIF was unsuccessful in $22 \%, 24 \%, 40.5 \%, 35.4 \%$, and $26.7 \%$ of patients over the 2 - to 10-year follow-up. However, the rate of unsuccessful TIF at 2 years was still the same at 10 years ( $\triangleright \mathbf{F i g} \mathbf{2 b}$ ).

Among the seven patients who underwent Nissen fundoplication, three showed a significant improvement in their GERrelated symptoms, while four did not. The mean GERD-HRQL scores before and 1 year after surgery in responders were $36 \pm$ 13 and $2.0 \pm 2.6$, respectively, compared to $57.5 \pm 20.7$ and $16.0 \pm 2.7$ in non-responders. At 2 years, only one of the four patients who had undergone surgery in the year after TIF completely stopped PPI therapy; at 5,7 , and 10 years, $57.1 \%$ of patients were still using PPI.

\section{Discussion}

The present observational study is the first assessing the clinical efficacy of the TIF 2.0 procedure for symptomatic gastroesophageal reflux disease for up to 10 years. The study covers a prospective series of consecutive patients with documented pathological GERD, treated by a single endoscopist in a single center. GER-related symptoms were assessed 2, 3, 5, 7, and 10 years after TIF, using GERD-HRQL questionnaires, heartburn, and regurgitation scores. Daily PPI dependence was also recorded for up to 10 years. The study was carried out in a carefully selected group of patients with chronic GERD who were not satisfied with medical therapy. Partial results at 2 years have already been published [11], so this study reports clinical results from 2 years after the TIF 2.0 procedure.

Our 2-year data agree with those reported in the only metaanalysis on TIF so far, which looked at the reports of five randomized control trials and 13 prospective observational studies (963 patients) published between 2007 and 2015 [8]. In that meta-analysis, the mean total satisfaction 6 months after TIF ranged between $58 \%$ and $86 \%$ (mean $69.15 \%$ ), and the total number of refluxes was significantly reduced, with outcomes comparable to that of surgery in the same period. Data on longer follow-up of patients who had undergone TIF were reported in the meta-analysis for three studies looking at 3-year outcomes in a limited number of cases [9-11]. The authors concluded that data on which to draw conclusions over the long term were lacking, but there was a tendency to restart PPI therapy in the majority of patients, though at lower doses than before TIF.

So far, four reports on 3-year outcomes have been published, with similar figures on symptom outcomes and PPI reduction or cessation. In the three studies examined in the meta-analysis, cessation of PPIs or halving their doses was reported in $74 \%, 74 \%$, and $84 \%$ of patients, while complete cessation of PPIs was reported in $42 \%, 65 \%$, and $53 \%$. A more recent study on 3-year outcomes showed better results, with elimination of troublesome regurgitation and all atypical symptoms in, respectively, $90 \%$ and $88 \%$ of patients, significantly lower mean percentages of time with $\mathrm{pH}<4$, healing of esophagitis and discontinuation of PPI therapy in $86 \%$ and $71 \%$ of patients [12]. Two other studies published in 2017 and 2018 on 44 and 60 patients reported elimination of symptoms and cessation of PPI therapy in $72.7 \%$ and $66 \%$ of patients at a median 59-month follow-up and at 5 years, respectively $[13,14]$. The total GERD health-related quality-of-life score improved decreasing from 22.2 to 6.8 at 5 years $(P<0.001)$ [14].

In the present study, symptomatic assessment with heartburn and regurgitation scores from the GERD-HRLQ questionnaire gave significantly lower scores than before treatment over the follow-up period. Clinical evaluation indicated that 2-, 3-, 5-, 7- and 10-year results remained close to those at 2 years and stable up to 10 years.

Daily high-dosage PPI dependence was eliminated in $86.7 \%$, $84.4 \%, 73.5 \%, 83.3 \%$, and $91.7 \%$ at $2,3,5,7$, and 10 years, respectively; the rates remained similar to those at 2 years; this provides further evidence of the lasting effect of TIF on symptoms and PPI usage. At 10 years, the rate of patients who stopped PPI or halved the PPI dose was even higher - $91.7 \%$; this improvement might possibly depend on the progressive scar-related fibrosis induced by the stitches over the years. 

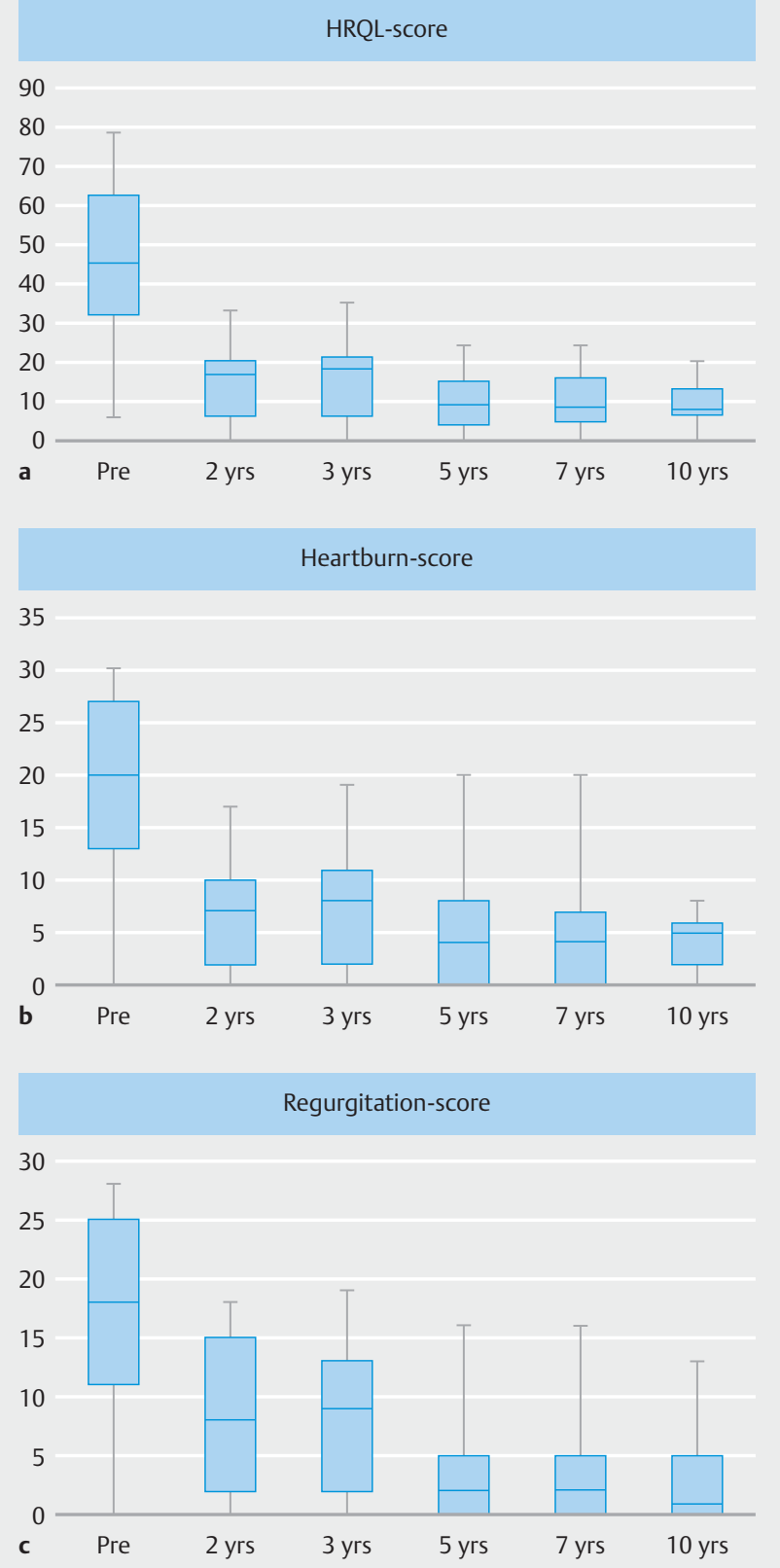

- Fig. 1 a Gastroesophageal reflux disease-Health-Related Qualityof-Life, $\mathbf{b}$ heartburn and $\mathbf{c}$ regurgitation scores when off proton pump inhibitor (PPI) therapy from baseline (T0) to 10-year followup. Data are reported as median and IQ range. HRQL, HealthRelated Quality-of-Life.

Considering only patients who completely stopped PPI therapy to be responders to TIF, the proportion of full responders was less satisfactory, although still significant, with rates of $55.6 \%, 53.3 \%, 41.2 \%, 45.8 \%$, and $41.7 \%$ at $2,3,5,7$, and 10 years, respectively. The complete response rate after TIF was slightly lower than at 2 years and was about $20 \%$ lower at 10 years; however, this reduction was not statistically significantly different $(P>0.05)$. Five-year outcomes in our study were less

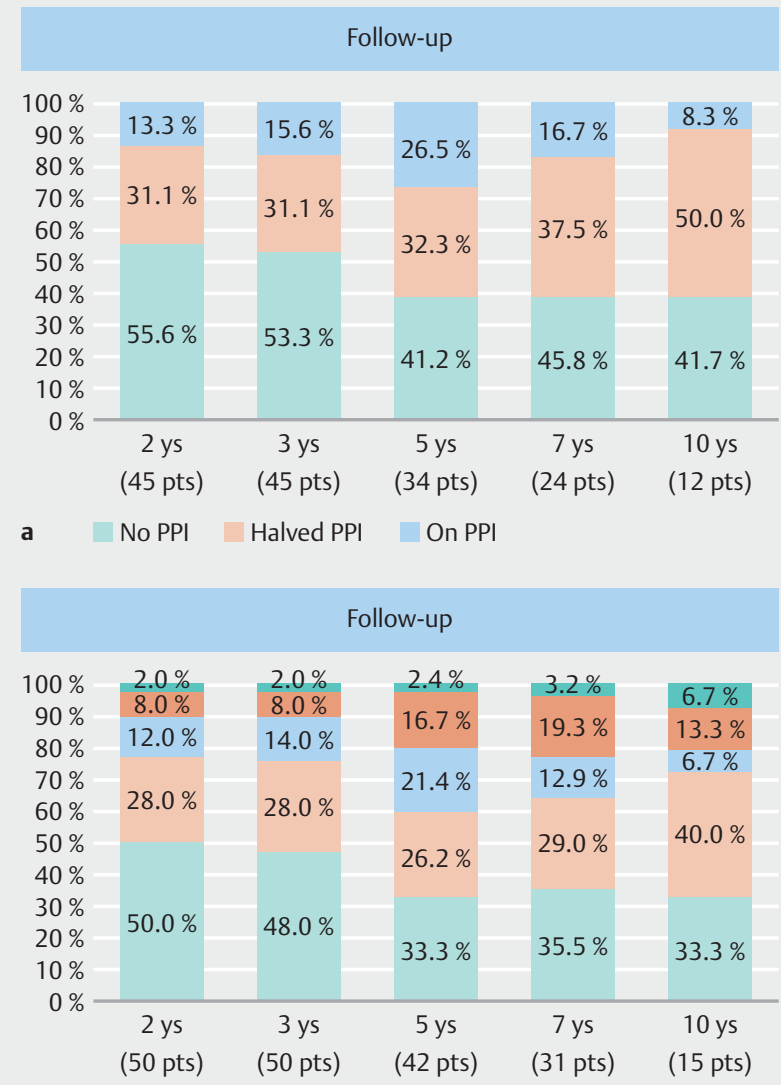

b $\quad$ No PPI Halved PPI $\backsim$ On PPI $\square$ Nissen FP $\square$ Complications

- Fig. 2 Symptomatic responses and proton pump inhibitor (PPI) use from 2 - to 10-year follow-up according to a per-protocol analysis and $\mathbf{b}$ intention-to-treat analysis. Data are reported as proportions on the $y$ axis. $P>0.05,3$ years vs 2 years; $P>0.05$, 5 years vs 3 years; $P>0.05,7$ years vs 5 years; $P>0.05,10$ years vs 7 years. Pts, patients; Ys, years; PPI, proton pump inhibitors; FP, fundoplication.

favorable than in the other 5-year outcomes study recently published (41.2\% vs.72.7\%) [13].

The limitation of the present study in the long-term followup is the limited number of patients clinically assessed at 7 and 10 years, without endoscopic and functional evaluations. Although only 24 and 12 patients had a 7- and 10-year post-TIF follow-up, the symptomatic curve over that period suggests that the results would not change much even with a larger number of cases.

Our results confirm that factors that negatively affect outcomes play a role only in the early postoperative period; patients who still respond 2 years after the operation enjoy a substantially unchanged response over the next few years.

In our published series [11], preoperative Hill grades III and IV and hiatal hernia larger than $2 \mathrm{~cm}$ at endoscopy, a small number of fasteners deployed (median $10 \pm 2$ ), and the presence of severe ineffective esophageal motility at manometry were all associated with a higher rate of early unsuccessful results. Ineffective esophageal motility is a problem seen in a heteroge- 
neous group with different manometric subsets and different symptom profiles, who might respond to the procedure in different ways [20].

The deployment of a larger number of fasteners (median 14 \pm 2 ) raised the probability of being a responder about four-fold, as stated in another paper [4]. The adoption of the rotational technique to create the new valve gave a $50 \%$ increase in successful outcomes too, according to other studies [4, 21].

These factors may all play roles in the unsatisfactory outcomes in patients who failed TIF and required Nissen fundoplication. Four of the seven patients who underwent surgery after TIF (57.1\%) still suffered GER symptoms. The low success rates after fundoplication in these selected patients with documented GERD, in whom functional heartburn was excluded, support the hypothesis that GERD-related symptoms might result from several different mechanisms, rather than just the impairment of the gastroesophageal junction. Although univariate and multivariate analysis showed that high preoperative GERD-HRQL scores, high PPI consumption, and age older than 50 years were the factors most closely associated with successful outcomes of transoral fundoplication [22], further data are still needed to clarify this point and help improve the selection of candidates for fundoplication, whether by TIF or laparoscopic surgery.

In our series, 2 - and 3-year post-TIF results were similar to those with surgical posterior partial (Toupet) or anterior partial fundoplication, but inferior to those reported in patients operated by posterior total fundoplication (Nissen) [23, 24], though without any of the surgery-related persistent side effects. On the other hand, 5-year post-TIF results were substantially similar to those reported with Nissen fundoplication. A nationwide register-based follow-up study on the use of PPI after anti-reflux surgery reported that, at 5 years, $57.5 \%$ and $29.5 \%$ of patients still took PPI or were daily PPI-dependent [25]. Our 5-year results also indicated that $58.8 \%$ and $26.5 \%$ of patients were still taking PPI or were daily PPI-dependent. In the Cochrane meta-analysis on Nissen fundoplication, the pooled analysis of long-term results showed recurrence or persistence of heartburn and reflux symptoms in $41.2 \%$ and $24.6 \%$ of cases, respectively, with persistent side effects in $14 \%$ up to $23 \%$ of cases [24].

There are very few reports of 10-year outcomes after surgical fundoplication but, again, results are substantially comparable with our TIF findings [25-27]. In a systematic review of partial responders to PPI who had undergone laparoscopic fundoplication, 10 years after the operation, 35.8\% reported heartburn and $29.1 \%$ regurgitation, with an $18.2 \%$ rate of acid-suppressive medication [26]. The nationwide registerbased follow-up study on the use of PPI after antireflux surgery showed that, at 10 years, $72.4 \%$ and $41.1 \%$ of patients were taking PPI or were daily PPI-dependent [25]. In our patients the proportions were $58.3 \%$ and $8.3 \%$.

TIF was associated with pneumothorax in 2 of the 51 cases (3.9\%). In both, this was the result of pleural perforation caused by insertion of the needle close to the lesser gastric curve. This complication should therefore be borne in mind when attempting to create a very tight valve. One meta-analysis reported that severe adverse events occurred in 19 out of 781 patients (2.4\%) [8]; this figure is lower than the $14 \%$ indicted in the Cochrane meta-analysis on Nissen fundoplication [24]. However, persistent side effects were reported after laparoscopic fundoplication in $5-19 \%$ of cases [24], while there were none after TIF.

In conclusion, in this clinical follow-up study, TIF 2.0 with EsophyX achieved lasting elimination or $50 \%$ reduction of daily PPI consumption in $73.5-91.7 \%$ of cases 2 to 10 years after the intervention, with no troublesome persisting procedure-related side effects. PPI medication was completely stopped in about $56 \%$ of patients at 2 years and $42 \%$ at 10 years, with no changes over the years. Unsuccessful outcomes after TIF result in recurrence of GERD symptoms within 6-12 months after the intervention, and thereafter clinical results remain substantially stable up to 10 years. Moreover, $57 \%$ of patients with TIF failure who underwent surgical fundoplication were still dissatisfied after surgery.

We found that 2-to 3-year outcomes were substantially similar to those reported for anterior surgical fundoplication, but worse than for total fundoplication; 5-and 10-year outcomes were also very similar to those for Nissen fundoplication.

We believe that our clinical findings are sufficient to confirm that TIF appears to offer a safe therapeutic option, as effective as surgery in the long-term, for carefully selected symptomatic GERD patients, with Hill grade I or II of the valve or hiatal hernia not longer than $2 \mathrm{~cm}$, who refuse life-long medical therapy or surgery, are intolerant to PPI, or have some risk of persistent post-surgical side effects.

\section{Competing interests}

None

References

[1] Cadière GB, Rajan A, Rquibate $M$ et al. Endoluminal fundoplication (ELF) - evolution of EsophyX ${ }^{\mathrm{TM}}$, a new surgical device for transoral surgery. Minim Invasive Ther Allied Technol 2006; 15: 348-355

[2] Cadiere GB, Rajan A, Germay O et al. Endoluminal fundoplication by a transoral device for the treatment of GERD: a feasibility study. Surg Endosc 2008; 22: $333-342$

[3] Jobe BA, O’Rourke RW, McMahon BP et al. Transoral endoscopic fundoplication in the treatment of gastroesophageal reflux disease. The anatomic and physiologic basis for reconstruction of the esophagogastric junction using a novel device. Ann Surg 2008; 248: 69-76

[4] Bell RC, Cadière GB. Transoral rotational esophagogastric fundoplication: technical, anatomical, and safety considerations. Surg Endosc 2011; 25: 2387-2399

[5] Spechler S]. Comparison of medical and surgical therapy for complicated gastroesophageal reflux disease in veterans. The Department of Veterans Affairs Gastroesophageal Reflux Disease Study Group. NEJM 1992; 326: $786-792$

[6] Herron DM, Swanstroem LL, Ramzi N et al. Factors predictive of dysphagia after laparoscopic Nissen fundoplication. Surg Endosc 1999; 13: $1180-1183$

[7] Catarci M, Gentileschi P, Papi C et al. Evidence-based appraisal of antireflux fundoplication. Ann Surg 2004; 239: 325-337 
[8] Huang X, Chen S, Zhao $\mathrm{H}$ et al. Efficacy of transoral incisionless fundoplication (TIF) for the treatment of GERD: a systematic review with meta-analysis. Surg Endosc 2017; 31: 1032 - 1044

[9] Witteman BPL, Strijkers R, de Vries E et al. Transoral incisionless fundoplication for treatment of gastro-esophageal reflux disease in clinical practice. Surg Endosc 2012; 26: 3307-3315

[10] Muls V, Eckardt AJ, Marchese M et al. Three-year results of a multicenter prospective study of transoral incisionless fundoplication. Surg Innov 2013; 20: 321 - 330

[11] Testoni PA, Testoni S, Mazzoleni G et al. Long-term efficacy of transoral incisionless fundoplication with EsophyX (TIF 2.0) and factors affecting outcomes in GERD patients followed for up to 6 years: a prospective single-center study. Surg Endosc 2015; 29: 2770 - 2780

[12] Trad K, Fox MA, Simoni G et al. Transoral fundoplication offers durable symptom control for chronic GERD: 3-year report from the TEMPO randomized trial with a crossover arm. Surg Endosc 2017; 31: 2498 2508

[13] Stefanidis G, Viazis N, Kotsikoros $\mathrm{N}$ et al. Long-term benefit of transoral incisionless fundoplication using the Esophyx device for the management of gastroesophageal reflux disease responsive to medical therapy. Dis Esophagus 2017; 30: 1-8

[14] Trad KS, Barnes WE, Prevou ER et al. The TEMPO trial at 5 years: transoral fundoplication (TIF 2.0) is safe, durable, and cost-effective. Surg Innov 2018; 25: 149-157

[15] Richter J, Kumar A, Lipka S et al. Efficacy of laparoscopic Nissen fundoplication vs transoral incisionless fundoplication or proton pump inhibitors in patients with gastro-esophageal reflux disease: a systematic review and network meta-analysis. Gastroenterology 2018; 154: 1298 - 1308.e7

[16] Drossman DA. The functional gastrointestinal disorders and the Rome III process. Gastroenterology 2006; 130: 1377-1390

[17] Velanovich V, Vallance SR, Gusz JR et al. Quality of life scale for gastroesophageal reflux disease. J Am Coll Surg 1996; 183: 217-224
[18] Raymond JM, Marquis P, Bechade D et al. Assessment of quality of life of patients with gastroesophageal reflux. Elaboration and validation of a specific questionnaire. Gastroenterol Clin Biol 1999; 23: 32 - 39

[19] Lundell LR, Dent J, Bennett JR et al. Endoscopic assessment of oesophagitis: clinical and functional correlates and further validation of the Los Angeles Classification. Gut 1999; 45: $172-180$

[20] Haack HG, Hansen RD, Malcolm A et al. Ineffective esophageal motility: manometric subsets exhibit different symptom profiles. World J Gastroenterol 2008; 14: 3719-3724

[21] Bell RC, Mavrelis PG, Barnes WE et al. A prospective multicenter registry of patients with chronic gastro-esophageal reflux disease receiving transoral incisionless fundoplication. | Am Coll Surg 2012; 215: $794-809$

[22] Bell RCW, Fox MA, Barnes WE et al. Univariate and multivariate analyses of preoperative factors influencing symptomatic outcomes of transoral fundoplication. Surg Endosc 2014; 28: 2949-2958

[23] Broeders JAJL, Mauritz FA, Ahmed Alì U et al. Systematic review and meta-analysis of laparoscopic Nissen (posterior total) versus Toupet (posterior partial) fundoplication for gastro-esophageal reflux disease. Br J Surg 2010; 97: 1318-1330

[24] Garg SK, Gurusamy KS. Laparoscopic fundoplication surgery versus medical management for gastro-oesophageal reflux disease (GORD) in adults. Cochrane Database Syst Rev 2015; 11: 1-35

[25] Loedrup A, Pottegard A, Hallas J et al. Use of proton pump inhibitors after antireflux surgery. A nationwide register-based follow-up study. Gut 2014; 63: $1544-1549$

[26] Lundell L, Bell M, Ruth M. Systematic review: laparoscopic fundoplication for gastro-esophageal reflux disease in partial responders to proton pump inhibitors. World J Gastroenterol 2014; 20: 804-813

[27] Kelly JJ, Watson DI, Chin KF et al. Laparoscopic Nissen fundoplication: clinical outcomes at 10 years. J Am Coll Surg 2007; 205: 570-575 